\title{
La polyphonie linguistique avec un regard sur l'approche scandinave
}

\author{
Henning Nølke \\ Université d'Aarhus \\ nolke@hum.au.dk
}

\section{Introduction}

Depuis Bally, la linguistique énonciative s'est développée comme une spécialité française. Aujourd'hui, elle occupe, sous ses différentes formes, une place importante dans le paysage linguistique français. Un de ses rejetons, devenu vigoureux avec l'âge, est la polyphonie linguistique.

Dans cet article, je présenterai d'abord un bref historique de l'évolution de la polyphonie linguistique, après quoi je proposerai une introduction à la version scandinave de cette théorie : la ScaPoLine (la théorie SCAndinave de la POlyphonie LINguistiquE).

Je tenterai enfin de montrer, sur quelques exemples, comment celle-ci est susceptible non seulement d'expliciter l'apport de la forme linguistique à l'interprétation du discours, mais aussi de servir d'outil heuristique pour des analyses de divers phénomènes linguistiques.

\section{Bref historique}

La notion de polyphonie a conquis la linguistique. Émanant d'études littéraires post-bakhtiniennes, le terme a envahi progressivement les analyses sémantiques ou pragmatiques. Sa popularité s'explique sans doute par la souplesse de la notion qui s'y rapporte. Le terme appelle une intuition immédiate. On a d'une certaine façon le sentiment que la langue est polyphonique. Tout discours semble en cacher un autre, simplement en raison du fait qu'il ne se produit jamais dans le vide, mais toujours dans un contexte qui appelle d'autres discours, déjà produits, à venir ou simplement imaginés. C'est la grande leçon que nous a apprise Bakhtine. Pourtant, dès que l'on tente d'expliciter en quoi consiste cette polyphonie, on se rend compte que la tâche est loin d'être simple. Un aperçu - même rapide - des emplois auxquels le terme a donné lieu, révèle immédiatement que la polyphonie joue à plusieurs niveaux d'analyse et qu'il y a presque autant de conceptions de cette notion que de linguistes qui s'en servent.

\subsection{Qu'est-ce que la polyphonie ?}

La première question qui se pose est donc la suivante: qu'est-ce que la polyphonie linguistique ? Intuitivement, tout texte fait parler plusieurs voix. Le locuteur se positionne par rapport à d'autres locuteurs, réels où simplement imaginés par lui, qu'il fait parler à travers son texte. Mais comment ces voix se manifestent-elles ? Comment peut-on les entendre ? Quelles sont leurs interrelations ? Et quelles sont leurs relations à la voix du locuteur? Autant de questions, autant de problèmes à résoudre.

Constatons tout d'abord que la polyphonie est un fait d'interprétation. La polyphonie fait partie du sens que l'allocutaire attribue au texte qu'il entend ou lit. Il arrive qu'un texte qui est polyphonique pour tel interlocuteur ne le soit pas pour tel autre. Mais il y a aussi des textes qui sont perçus comme polyphoniques par tout un chacun (du moins après réflexion) indépendamment de leurs contextes. La forme linguistique semble donc également jouer un rôle important pour la genèse de la polyphonie. Mais comment?

Un autre problème découle du fait que les mêmes phénomènes ne sont pas perçus comme polyphoniques par tous les chercheurs. Pour certains, la polyphonie est un phénomène textuel par excellence. Ainsi, pour le grand précurseur, Michaël Bakhtine, la polyphonie caractérise un type de texte: le roman 
polyphonique. Celui-ci se caractérise par le fait que le héros est à égalité avec l'auteur (Bakhtine 1970 : 53). Pour d'autres, la polyphonie se retrouve au micro-niveau de l'énoncé, chaque énoncé étant susceptible d'être interprété comme un discours cristallisé. C'est l'idée défendue par Ducrot.

Confronté à ce double problème, il faudra trancher. La seule voie viable me semble être de «diviser chacune des difficultés [...] en autant de parcelles qu'il se pourra, et qu'il sera requis pour les mieux résoudre ", quitte à tenter une synthèse ultérieurement si les éléments élaborés s'y prêtent. C'est dans cet esprit que j'ai opté pour une approche qui se concentre sur le niveau de complexité de l'énoncé, et sur le codage linguistique des effets polyphoniques qu'on peut y observer. Mon objet d'étude sera la polyphonie en langue, conçue comme le produit des éléments de la langue susceptibles de favoriser une certaine lecture polyphonique de la parole. On verra que cette restriction soulève tout de suite de nouveaux problèmes : en quel sens un élément de la langue peut-il favoriser une certaine lecture ? Quels sont ces éléments et comment (par quelles méthodes) peut-on les trouver et les décrire ? Et plus généralement, quel est le rapport entre le fonctionnement de ces éléments, le contexte (au sens large) et le processus d'interprétation, les rapports entre langue, parole et réception?

\subsection{L'approche ducrotienne}

L'idée de la présence de plusieurs «voix» dans certains énoncés se trouve déjà, sous forme embryonnaire, chez des auteurs comme Banfield (1979), Desclés (1976) et Plénat (1979), mais c'est le grand mérite d'Oswald Ducrot d'avoir introduit la notion de polyphonie dans les études linguistiques $(1982 ; 1984: \mathrm{ch} . \text { VIII })^{1}$. L'originalité de son approche réside dans la scission du sujet parlant au niveau de l'énoncé même. On sait qu'il a introduit une distinction systématique entre le locuteur et les énonciateurs. Le locuteur est celui qui, selon le sens de l'énoncé, est responsable de l'énonciation. Il peut mettre en scène divers énonciateurs qui présentent différents points de vue. Il peut s'associer à certains énonciateurs tout en se dissociant de certains autres. Il est important de souligner que tous ces 'êtres discursifs' sont des êtres abstraits. Le rapport à l'être parlant réel n'intéresse pas Ducrot.

La polyphonie de Ducrot a eu une grande influence en sémantique française. Il n'a cependant jamais développé lui-même une véritable théorie de la polyphonie et sa terminologie change légèrement d'un ouvrage ou d'un article à l'autre. Notamment le statut exact des énonciateurs est resté difficile à cerner, ce qui a donné lieu à de multiples définitions ou redéfinitions chez les linguistes qui se sont inspirés de Ducrot pour reprendre la distinction entre locuteur et énonciateurs. Dans son article de 2001, qui a été rédigé comme une réponse à l'approche scandinave (la ScaPoLine), il reprend cette question pour donner des précisions. Il liste trois conceptions possibles et opte pour la troisième, selon laquelle les énonciateurs sont des locuteurs virtuels et leurs points de vue des " paroles virtuelles, d'un discours envisagé sans que personne ne soit censé l'avoir prononcé, ni tel quel ni sous une autre forme » $(2001: 38)^{2}$. Ces discours virtuels (cristallisés) constituent le « contenu » de l'énoncé (1984: 224), et les énonciateurs s'en servent pour «constituer une représentation de la réalité »(2001: 38-39). Par ce moyen, les énonciateurs « voient » les choses à travers les mots. La fonction du locuteur est alors, pour Ducrot, de communiquer les discours des énonciateurs en prenant parti face aux différentes représentations qu'ils constituent (ibid. : 39).

Sauf erreur, le but de Ducrot semble être d'axer son étude sur la polyphonie en langue. Son ambition est de rendre compte des interprétations virtuelles de tout énoncé à partir de sa forme linguistique. Son objet d'étude est la langue au sens de Saussure, et plus particulièrement les traces que laisse l'énonciation dans la forme linguistique. Il s'intéresse au codage linguistique de la polyphonie au niveau du système et en relation à un discours idéalisé, à un discours associé au contexte que détermine la forme linguistique en soi.

\subsection{Polyphonie et dialogisme}

Si Ducrot s'est expressément concentré sur la polyphonie en langue, d'autres chercheurs, inspirés par ses travaux ou par ceux de Bakhtine se sont plutôt intéressés à la polyphonie en discours. La position prise 
n'est cependant pas toujours claire et beaucoup d'analyses qui se réclament de l'approche ducrotienne utilisent plutôt les notions développées par cet auteur de manière non formelle et parfois même impressionniste, ce qui n'empêche pas qu'ils réussissent souvent à découvrir et à décrire des effets de sens polyphonique fort intéressants et utiles pour notre compréhension de la polyphonie discursive. En effet, il ne faut pas oublier qu'indépendamment de nos choix théoriques, la polyphonie manifestée dans les textes ou dans le discours reste notre seul observable.

Deux approches méritent des remarques particulières : le modèle de l'organisation du discours développé à Genève autour d'Eddy Roulet et la praxématique développée à Montpellier. Ces deux approches représentent de véritables élaborations théoriques qui se distinguent sur plusieurs points de la polyphonie « ducrotienne » (et, partant, de la ScaPoLine). Ainsi le domaine d'application du modèle de Genève est-il plus étendu. A l'opposé de Ducrot, qui s'en tient à l'analyse d'énoncés ou de brefs segments isolés, les Genevois situent la description polyphonique dans un cadre (modulaire) plus large en insistant sur ses rapports à d'autres aspects de l'organisation du discours. Pour eux, la polyphonie est une notion complexe qui se construit à partir de notions plus primitives. D'autre part, le domaine conceptuel de cette approche est plus restreint et moins abstrait. Se centrant sur le traitement de diverses formes de discours représenté, elle n'a pas recours aux «énonciateurs » ou aux «points de vue » de Ducrot. Pour les Genevois, il y a polyphonie seulement s'il y a plusieurs locuteurs - réels ou présentés.

Le dialogisme développé par Jacques Bres et ses collègues dans le cadre de la praxématique s'inspire directement des travaux de Bakhtine. Cette théorie linguistique a été développée indépendamment de la théorie polyphonique. Il s'avère néanmoins qu'il existe de nombreuses analogies entre les deux approches qui, dans une large mesure, traitent des mêmes phénomènes de sens, et les dernières années ont vu paraître plusieurs articles traitant des ressemblances et différences entre les deux approches (voir par exemple Bres 2005, Dendale 2007, Nølke 2006a). On peut notamment remarquer une analogie parfois frappante entre le dialogisme de la praxématique et la ScaPoLine, théorie qui se veut être une tentative visant à élaborer une théorie formalisée basée sur les idées originales d'Anscombre et de Ducrot.

\section{ScaPoLine 2008}

Je voudrais donc proposer une introduction à la théorie SCAndinave de la POlyphonie LINguistiquE. Fortement inspiré par les travaux d'Anscombre et Ducrot, je développe, avec des collègues, depuis plus de vingt ans une théorie linguistique de la polyphonie, baptisée la ScaPoLine il y a une dizaine d'années. Quoique fidèle aux idées essentielles de l'approche ducrotienne, la ScaPoLine a pris un certain nombre de décisions théoriques qui la distingue. Les différences ne s'expriment pas par une autre conception de la polyphonie linguistique mais par des buts différents. Notre ambition est de créer une théorie formalisée qui soit en mesure de prévoir et de préciser les contraintes proprement linguistiques qui régissent l'interprétation polyphonique. Notre espoir est que cette insistance sur l'ancrage formel nous permette de faire de la ScaPoLine un appareil heuristique rendant possible des analyses opératoires, non seulement des énoncés individuels, mais aussi de fragments de textes composés de plusieurs énoncés.

\subsection{Fondement conceptuel}

Suivant Ducrot, nous partons de l'observation que la présence de certaines expressions linguistiques dans le discours semble entraîner une lecture polyphonique des énoncés qui les abritent. Rappelons l'exemple « canonique » (cf. note 1$)$ :

(1) Ce mur n'est pas blanc

Dans cet énoncé, on a nettement l'impression que deux points de vue (incompatibles) cohabitent :

$$
\begin{aligned}
& \operatorname{pdv}_{1}: \text { 'ce mur est blanc' } \\
& \operatorname{pdv}_{2}: \operatorname{pdv}_{1} \text { est injustifié' }
\end{aligned}
$$


Si le locuteur s'est servi de la négation, c'est en effet parce que quelqu'un pense (ou pourrait penser) que le mur est blanc $\left(\mathrm{pdv}_{1}\right)$, ce qui est contraire à l'opinion du locuteur $\left(\mathrm{pdv}_{2}\right)$. Notons qu'alors que pdv 2 (qui prend le contre-pied de $\mathrm{pdv}_{1}$ ) est forcément le point de vue du locuteur (ce qu'on voit par le fait que celuici ne peut pas - dans un discours cohérent - nier avoir ce point de vue), on ne peut pas déduire du seul énoncé qui est responsable du premier pdv.

Ce sont des observations de ce genre qui ont inspiré la théorisation linguistique de la polyphonie. L'important est alors que l'existence de ces deux points de vue est codée dans les matériaux linguistiques mêmes par la présence de la négation ne...pas. En effet, elle se révèle dans la nature des enchaînements possibles :

Ce mur n'est pas blanc.

a. - Je le sais.

b. (...), ce que regrette mon voisin.

a. - Pourquoi le serait-il ?

b. (...), ce que croit mon voisin.

c. (...) Au contraire, il est tout noir.

On voit que les réactions (monologales comme dialogales) dans (2) renvoient au point de vue (négatif) du locuteur, alors que celles de (3) (monologales comme dialogales) enchaînent sur le point de vue positif (sous-jacent) véhiculé à travers (1). Il est remarquable que même les enchaînements monologaux dans (3) s'attachent à ce dernier point de vue, dont le locuteur se distancie explicitement. Cette double possibilité d'enchaînement n'existerait pas sans la présence de la négation syntaxique.

Cette manière d'argumenter illustre d'ailleurs un trait essentiel de la ScaPoLine : celle-ci traite des phénomènes qui sont engendrés dans la langue, en principe indépendamment de son emploi. Son objet d'étude réside dans les instructions que pourvoit la langue pour l'interprétation polyphonique des énoncés. C'est dans ce sens que la théorie polyphonique est une théorie sémantique, discursive, structuraliste et instructionnelle. Ces instructions peuvent être plus ou moins précises. Dans l'énoncé de (1), l'instruction consiste à faire comprendre à l'allocutaire que deux points de vue contradictoires sont en jeu, l'un positif, l'autre négatif, et que le locuteur s'associe à ce dernier. Mais elle n'exprime rien quant à la source du point de vue positif. L'output linguistique au sens restreint sera donc à concevoir comme une structure sémantique renfermant quelques variables. Dans notre cas spécifique, la valeur d'une des variables est précisée, alors que celle de l'autre reste tout à fait ouverte. Dans le processus interprétatif, le destinataire physique cherchera alors automatiquement (et inconsciemment) à découvrir l'identité de celui qui est responsable de l'autre point de vue (en l'occurrence pdv $v_{1}$ ).

\subsection{Le locuteur Axiomatique (L-A)}

$\mathrm{Si}$, en dernière instance, l'objet d'étude de la ScaPoLine est la structure sémantique, cette étude passe donc par l'examen de l'énoncé que, suivant Ducrot, nous concevons comme une image de l'énonciation ${ }^{3}$. C'est un axiome de la théorie que toute énonciation a un locuteur : le Locuteur Axiomatique (abrégé en LA ou tout simplement appelé le locuteur) qui est construit dans l'énonciation par la langue. Par extension, le texte, composé d'énoncés, est conçu comme le produit du discours : c'est une image "gelée » du discours créée par L-A. C'est L-A qui assure l'ancrage du texte dans le monde social. Il est une image particulière du sujet parlant (ou écrivant). On peut dire qu'il est un masque que se donne l'homme en parlant ou en écrivant. Cette construction de L-A est probablement plus ou moins inconsciente dans la vie quotidienne où le masque tend à être adapté à la situation discursive particulière : ce n'est pas le même LA que l'on construit pour parler à son chef ou à ses enfants. On peut également imaginer que cette construction est beaucoup plus consciente et sophistiquée dans les textes littéraires où l'auteur physique construit souvent soigneusement L-A : l'auteur du texte. Si intéressante que soit l'étude de cette relation entre le sujet parlant et L-A, elle dépasserait cependant le cadre de la ScaPoLine. 
Or, c'est un trait constitutif de la langue que de permettre - dans son emploi - la présence de traces de l'activité concrète du Locuteur Axiomatique. Cette propriété s'explique sans doute par le dialogisme inhérent à la langue et ces traces font partie du domaine d'étude de la ScaPoLine. Ainsi L-A peut-il construire plusieurs types d'images de lui-même ou plutôt des divers rôles qu'il est susceptible de jouer dans ses énoncés. On distinguera deux types principaux : LOC, qui est une image de L-A dans son rôle de constructeur de l'énonciation, et différentes images de lui comme source de points de vue. De plus, L-A construit sans cesse des images de toutes les autres «personnes » qui peuplent le discours telles qu'il désire les représenter. Parmi ces images, on notera en particulier les êtres discursifs qui font partie des éléments fondamentaux de la ScaPoLine.

\subsection{Les éléments fondamentaux}

En vue de préciser les niveaux d'analyse, nous distinguerons la structure polyphonique (abrégée structure-p) de la configuration polyphonique (abrégée configuration). La structure-p se situe au niveau de la langue (ou de la phrase), et c'est la raison pour laquelle elle ne se découvre pas par une étude des interprétations ou des emplois possibles des énoncés, mais seulement par un examen des (co)textes auxquels ceux-ci sont susceptibles de s'intégrer. En revanche, la structure-p fournit des instructions relatives à l'interprétation polyphonique de l'énoncé de la phrase, ou plus précisément aux interprétations possibles de l'énoncé. Le résultat de ce procédé est la création de la configuration polyphonique, qui fait partie de l'interprétation que fait l'allocutaire du texte auquel il est confronté pris dans sa globalité. Notre objet d'étude est la structure-p, mais n'ayant aucun accès direct à la langue, il nous faudra d'abord étudier la configuration qui est notre seul observable. Selon la ScaPoLine, la configuration se compose de quatre éléments fondamentaux :

- Le locuteur-en tant que constructeur (LOC) assume la responsabilité de l'énonciation.

- Les points de vue (pdv) sont des entités sémantiques porteuses d'une source qui est dite avoir le $\mathrm{pdv}^{4}$. Les sources sont des variables. Elles correspondent aux énonciateurs d'Anscombre et Ducrot.

- Les êtres discursifs (ê-d) sont des entités sémantiques susceptibles de saturer les sources.

- Les liens énonciatifs (liens) relient les ê-d aux pdv.

Les quatre éléments de la configuration sont tous susceptibles d'être codés dans la langue et, partant, de faire partie de la structure polyphonique, mais ils ne le sont pas forcément.

Considérons donc rapidement chacun de ces quatre éléments fondamentaux de la ScaPoLine.

\subsection{LOC}

L'élément LOC est nouveau par rapport à la polyphonie de Ducrot chez qui le locuteur semble couvrir plusieurs aspects. Soulignons d'emblée que LOC n'est pas un ê-d selon nos définitions : il n'est pas à même d'être source de pdv. Sa fonction comme constructeur de l'énonciation laisse néanmoins certaines traces linguistiques. Ainsi, c'est lui qui se trouve au centre de convergence des marques déictiques - c'est lui qui est le moi de moi-ici-maintenant. Il s'ensuit qu'il est directement responsable des temps grammaticaux qui renvoient à ce centre. Considérons l'exemple sous (4) :

(4) Pierre a dit qu'il viendrait.

Dans cet énoncé, le conditionnel fonctionne comme un futur du passé. On verra que le futur est celui de Pierre, mais le passé est celui du locuteur. En effet le discours rapporté a eu lieu dans le passé par rapport au centre déictique de LOC.

LOC peut aussi commenter son énonciation en la modalisant. Tout élément métalinguistique ou métatextuel (métadiscursif?) comme voir plus loin, d'une part...d'autre part porte sur la construction même de l'énonciation reflétant ainsi la présence de LOC. Il en va de même des adverbes d'énonciation, tels sans rire, si j'ose dire, qui modalisent différents aspects de l'acte illocutoire. En un certain sens, en se 
servant de ces adverbes, LOC se construit quand même comme source de pdv, à savoir des pdv qui portent sur sa propre énonciation ${ }^{5}$.

Précisons enfin qu'en tant que constructeur, c'est LOC qui assume la responsabilité des actes illocutoires et argumentatifs construits par l'énoncé.

Maître souverain de son énonciation, LOC est également en mesure de construire la représentation d'un autre locuteur. Cet 'Autre' peut être lui-même à un autre moment, il peut s'agir de l'allocutaire ou d'une tierce personne. LOC peut choisir de se contenter de représenter (certains de) ses pdv comme il le fait dans le Discours Indirect dont (4) est un exemple. Dans ce cas, le locuteur représenté, LR, sera construit comme un ê-d. Plus intéressante est peut-être l'autre possibilité : LOC peut aussi, par le jeu d'une forme de discours direct, représenter l'autre locuteur avec toutes ses propriétés de locuteur comme dans (5)

(5) Pierre a dit : « Je reviendrai ».

Il s'agit alors d'une instance de mimétisme ${ }^{6}$. LOC emboîte le discours de l'Autre dans son propre discours, prétendant ne pas y toucher mais seulement le reproduire. L'autre locuteur n'est plus construit comme un ê-d, mais comme un locuteur entier : il est un LOC mimé. On peut en distinguer trois instances: $\mathrm{LOC}_{\mathrm{t}}$, ALLOC, TIERS, selon qu'il s'agit du locuteur lui-même à un autre moment $\mathrm{t}$, de l'allocutaire ou d'une troisième personne.

\subsection{Les points de vue (pdv)}

La confusion terminologique qui caractérise les emplois de ce terme est particulièrement prononcée. Très souvent différents auteurs discutent des pdv (ou des PDV !) comme s'ils parlaient de la même chose. Or, à y regarder de plus près, il s'avère presque toujours qu'il ne s'agit pas exactement du même phénomène. La confusion s'explique sans doute par le fait que le terme appartient à la langue quotidienne, avant d'avoir reçu différentes acceptions plus ou moins précises, chez les littéraires aussi bien que chez les linguistes. Il a évidemment servi à mettre en évidence des phénomènes importants. Ainsi, les travaux de Genette, de Fauconnier ou de Rabattel, pour ne mentionner que quelques auteurs ayant défini précisément leurs pdv, nous ont beaucoup fait progresser dans nos propres recherches. Mais la confusion trouble gravement l'échange des idées. Ce domaine mériterait en effet une étude notionnelle comparative, analogue à celle qu'ont menée Coltier \& Dendale (2005) sur la notion de 'prise en charge'.

Dans le cadre de la ScaPoLine, nous avons proposé une définition des pdv selon laquelle leur forme générale est :

$$
\text { [ X ] (JUGE (p)) }
$$

où $X$ symbolise la source, $J U G E$ le jugement et $p$ le contenu. La source correspond aux énonciateurs de Ducrot, la différence étant que pour nous, elle appelle une saturation, si possible. Lors du processus interprétatif de l'énoncé, l'interprète cherche (automatiquement) à la saturer par un être discursif. Ainsi, les êtres discursifs deviennent-ils des éléments centraux de notre théorie (cf. 3.6).

Nous distinguerons trois types de pdv, à savoir les pdv simples et deux types de pdv complexes : hiérarchiques et relationnels. L'énoncé de (7) ne véhicule qu'un pdv simple :

$$
\begin{aligned}
& \text { Il fait beau. } \\
& \text { pdv : [ X ] ( VRAI ('il fait beau') ) }
\end{aligned}
$$

(7) est dit monophonique au niveau de la langue, sa structure ne contenant qu'un pdv. Par défaut, le locuteur est source de ce pdv.

Les pdv hiérarchiques se composent de points de vue simples ou complexes organisés selon une structuration hiérarchique. Les pdv hiérarchiques permettent de faire porter des jugements (extérieurs) sur d'autres jugements. L'énoncé de (1) en est un exemple :

$$
\begin{aligned}
& \text { Ce mur n'est pas blanc. } \\
& \operatorname{pdv}_{1}:[\mathrm{X}] \text { ( VRAI ('ce mur est blanc')) }
\end{aligned}
$$




$$
\left.\operatorname{pdv}_{2}:\left[1_{0}\right] \text { ( INJUSTIFIÉ }\left(\operatorname{pdv}_{1}\right)\right)
$$

Les pdv relationnels, enfin, relient des pdv simples ou complexes entre eux. On aura notamment des pdv relationnels dans les phrases renfermant des connecteurs comme dans (8) :

$$
\text { Il fait beau donc Pierre se promène. }
$$

qui véhicule trois pdv dont un relationnel :

$$
\begin{aligned}
& \operatorname{pdv}_{1}:[\mathrm{X}](\operatorname{VRAI}(\mathrm{p})) \\
& \operatorname{pdv}_{2}:\left[1_{0}\right](\operatorname{VRAI}(\mathrm{q})) \\
& \operatorname{pdv}_{3}:\left[1_{0}\right](\text { TOP }(\operatorname{si} p \text { alors } q))
\end{aligned}
$$

où $\mathrm{p}=$ 'il fait beau' et $\mathrm{q}=$ 'Pierre se promène'. TOP signifie 'il est généralement vrai que'.

\subsection{Les êtres discursifs (ê-d)}

L'introduction des êtres discursifs comme terme formel est propre à la ScaPoLine. Ducrot en parle, mais pour lui ce terme sert plutôt à distinguer les "êtres linguistiques" de leurs prétendus homologues physiques. L'introduction des ê-d est une conséquence immédiate de notre désir d'assurer l'ancrage formel de l'interprétation polyphonique concrète. Nous concevons les ê-d comme des images des participants au discours ou des autres personnages qui s'y trouvent mentionnés. Cette idée d'imagerie est une tentative de capter l'intuition qui me semble sous-tendre toute l'idée de la polyphonie linguistique : les «voix » émanent de personnages qui sont en quelque sorte des images que le locuteur présente à travers son énonciation, des référents discursifs qui peuplent l'univers réel ou fictif auquel il réfère.

Les ê-d se regroupent en trois classes selon les référents discursifs dont ils sont des images : les images de la première personne (L-A), de la deuxième personne (l'allocutaire) et des troisièmes personnes. Les trois types d'images sont tous susceptibles de figurer dans la structure-p : ils peuvent tous laisser des traces linguistiques. L'énoncé de (9) est un exemple d'une trace de la deuxième personne :

Dis-moi ce que j'ai mangé ce matin puisque tu sais tout.

Selon l'analyse de Ducrot, généralement acceptée, puisque introduit un pdv dont l'allocutaire est tenu responsable. Soulignons que LOC est libre de construire les images tout à sa guise. Les ê-d ne sont que ses marionnettes. Si (9) reçoit probablement une lecture ironique, c'est parce que LOC a choisi de construire une image absurde de son allocutaire en présentant celui-ci comme une personne qui prétend tout savoir.

Les ê-d peuvent être présentés comme des êtres qui ont effectivement produit des pdv, par exemple à travers des énonciations, ce sont des êtres discursifs d'énoncé ${ }^{7}$, ou simplement comme des ê-d ayant des pdv sans référence à aucune production de ce pdv, ce sont des êtres discursifs textuels ${ }^{8}$. Le locuteur joue un rôle privilégié dans ce jeu pour autant qu'il puisse assumer la responsabilité d'un pdv construit dans l'énonciation actuelle. Cette image de L-A est LE locuteur de l'énoncé. Il correspond au locuteur en-tantque-tel de Ducrot.

Nous avons ainsi les types suivants d'ê-d :

\section{Êtres discursifs}

- Première personne : $\mathrm{L}, \mathrm{l}_{0}, \mathrm{l}_{\mathrm{t}}(\mathrm{t} \neq 0)$

- Deuxième personne : $\mathrm{A}, \mathrm{a}_{\mathrm{t}}(\mathrm{t} \neq 0)$

- Troisième personne (voir ci-dessous)

Les majuscules symbolisent les ê-d textuels et les minuscules les ê-d d'énoncé, l'indice indiquant le moment où l'ê-d a produit le pdv en question. Les ê-d de la troisième personne sont appelés les tiers. Ceux-ci n'ont pas encore fait l'objet d'études avancées dans le cadre de la ScaPoLine. Nous comptons les types suivants :

- Les tiers individuels : $\mathrm{T}, \tau_{\mathrm{t}}(\mathrm{t} \neq 0)$ 
- Les tiers collectifs :

- Les tiers hétérogènes (variantes de $\mathrm{ON}$ polyphonique)

○ Les tiers homogènes (LOI, idées reçues, ...)

Les tiers individuels correspondent aux ê-d des première et deuxième personnes, à ces tiers individuels s'ajoutent les tiers collectifs. LOC est en effet en mesure de construire des pdv dont des collectifs sont tenus responsables. C'est le cas des présuppositions, par exemple. L'énoncé de (10) nous en donne un exemple :

(10) Pierre a cessé de fumer.

Suivant Ducrot (qui s'est inspiré de Berrendonner), nous analysons le présupposé aspectuel véhiculé par (10) comme un pdv dont ON assume la responsabilité :

$$
\begin{aligned}
& \operatorname{pdv}_{1}:[\mathrm{ON}](\text { VRAI ('Pierre fumait autrefois')) } \\
& \operatorname{pdv}_{2}:\left[1_{0}\right] \text { ( VRAI ('Pierre ne fume pas actuellement')) }
\end{aligned}
$$

ON est un tiers hétérogène incluant $\mathrm{L}$ et $\mathrm{A}^{9}$. C'est d'ailleurs cet exemple de la présupposition qui a inspiré à Ducrot d'introduire sa distinction entre le locuteur en-tant-que-tel et le locuteur en tant qu'être-dumonde, distinction qui correspond grosso modo à la nôtre entre le locuteur de l'énoncé, $1_{0}$, et le locuteur textuel, L.

La présupposition introduit ainsi (indirectement) une polyphonie entre deux images du locuteur : c'est la polyphonie interne. La présupposition n'est pas le seul élément linguistique codant la polyphonie interne. Ainsi ai-je argumenté ailleurs (Nølke 1985) en faveur d'une analyse du subjonctif selon laquelle ce mode marque la polyphonie interne, et même au sens strict :

(11) Je comprends que Pierre soit parti.

Selon cette analyse, (11) véhicule les deux pdv sous (11') :

(11') $\quad \operatorname{pdv}_{1}:[\mathrm{L}]($ VRAI ('Pierre est parti')) $\operatorname{pdv}_{2}:\left[1_{0}\right]\left(\right.$ COMPRENDRE $\left(\right.$ PDV $\left.\left._{1}\right)\right)$

COMPRENDRE se lira 'avoir compréhension pour'. Si nous parlons de la polyphonie interne au sens strict, c'est parce qu'elle s'effectue uniquement entre deux images du locuteur. La présupposition fait aussi intervenir d'autres ê-d, indiquant ainsi à la fois la polyphonie interne et la polyphonie externe.

Notons enfin que les exemples (1) (la négation) et (9) (puisque) sont des exemples de polyphonie externe au sens strict pour autant que ces exemples véhiculent deux pdv dont l'un est assumé par $1_{0}$, l'autre par un ê-d autre que la première personne.

Dans $\S 4$ je présenterai quelques exemples d'autres combinaisons d'êtres discursifs.

\subsection{Les liens énonciatifs (liens)}

Mais d'abord il nous faudra présenter le dernier élément fondamental de la configuration. Les liens (énonciatifs) établissent des relations entre les différents ê-d et les pdv. Nous opérons une distinction primaire entre le lien de responsabilité et les liens de non-responsabilité (dont il y a plusieurs types). Par définition, l'ê-d qui est (pris pour) source d'un pdv donné engage un lien de responsabilité avec ce pdv. Il importe de souligner qu'être source de $\mathrm{pdv}_{\mathrm{i}}$ et assumer la responsabilité de $\mathrm{pdv}_{\mathrm{i}}$ revient au même pour nous. Tout ê-d - et notamment toute image du locuteur - est susceptible d'être relié à un pdv dont il n'est pas source, par un lien de non-responsabilité.

Sera introduite ensuite une catégorisation secondaire des liens de non-responsabilité qui les divise en liens réfutatifs et liens non réfutatifs ${ }^{10}$. Nous arriverons ainsi à la classification résumée dans la figure $1^{11}$ : 
Figure 1

\begin{tabular}{|l|c|c|}
\hline \multirow{2}{*}{ LIENS DE RESPONSABILITÉ } & \multicolumn{2}{|c|}{ LIENS DE NON-RESPONSABILITÉ } \\
\cline { 2 - 3 } & liens non réfutatifs & liens réfutatifs \\
\hline
\end{tabular}

Si un ê-d, X, entretient un lien de responsabilité avec $\mathrm{pdv}_{\mathrm{i}}$, cela signifie que :

- le contenu propositionnel de $\mathrm{pdv}_{\mathrm{i}}$ (s'il y en a un) est vrai pour X

- X a eu l'idée d'avancer $\mathrm{pdv}_{\mathrm{i}}$

- $\mathrm{X}$ prend à son compte toutes les opinions et systèmes de valeurs qui émanent $\mathrm{de}_{\mathrm{pdv}}-\mathrm{y}$ compris son argumentativité

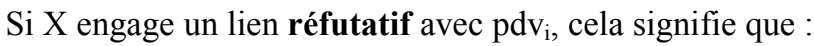

- le contenu propositionnel de $\mathrm{pdv}_{\mathrm{i}}$ (s'il y en a un) est faux pour X

- s'il n'y a pas de contenu propositionnel $\mathrm{pdv}_{\mathrm{i}}, \mathrm{X}$ considère que ce pdv est erroné

Si X ni n'assume la responsabilité de $\mathrm{pdv}_{\mathrm{i}}$ ni ne réfute $\mathrm{pdv}_{\mathrm{i}}$ il l'accorde à un certain degré. Ce degré peut varier et il semble probable que les liens de non-responsabilité et de non-réfutation s'organisent sur une échelle partant des liens qui sont tout près de la réfutation et orientée vers les liens d'accord fort ${ }^{12}$. Différents éléments linguistiques semblent susceptibles d'indiquer l'emplacement d'un lien donné sur cette échelle. Selon cette idée, certes indiquerait, dans les structures concessives, un lien tout près du pôle 'presque-responsable' sans pour autant franchir la frontière, tandis que l'adverbe peut-être, par exemple, indiquerait un emplacement plus éloigné de ce pôle. Considérons les exemples suivants :

(12) $: 0$ Certes tu es resté chez toi toute la nuit, mais je t'ai vu au café à minuit.

(13) ? Peut-être que tu es resté chez toi toute la nuit, mais je t'ai vu au café à minuit.

Dans les deux exemples, $1_{0}$ accorde le pdv concédé sans en assumer la responsabilité. Or dans (12), certes indique qu'il le considère comme vrai ; il le prend en charge selon une définition répandue de la prise en charge $^{13}$. Le lien codé par certes partage ainsi une des trois propriétés définitoires du lien de responsabilité, ce qui le place tout près du pôle positif. C'est la prise en charge qui rend l'énoncé de (12) fortement bizarre jusqu'au point d'être non acceptable, puisqu'elle entraîne une incompatibilité entre la partie concédée et la partie posée. L'énoncé de (13) n'entraîne pas la même bizarrerie, peut-être admettant la possibilité que la proposition concédée soit fausse.

Je termine cette brève discussion des liens énonciatifs en soulignant qu'il reste bien des problèmes épineux à résoudre dans ce domaine. Ainsi la critique avancée par Coltier \& Dendale a très bien montré que le rapport entre la composante JUGE des pdv et les liens est loin d'être clair. J'espère pouvoir revenir sur les relations entre les divers concepts invoqués dans un travail ultérieur.

\subsection{Bilan}

Rappelons les éléments de la configuration polyphonique sous forme schématique : 
Figure 2

\begin{tabular}{|c|c|c|c|c|c|}
\hline $\begin{array}{l}\text { L-A (le } \\
\text { locuteur) }\end{array}$ & \multicolumn{5}{|c|}{ Le Locuteur Axiomatique } \\
\hline LOC & \multicolumn{5}{|c|}{ Le locuteur en tant-que-constructeur } \\
\hline \multirow{3}{*}{$\begin{array}{l}\text { Les points de } \\
\text { vue, } \\
\text { pdv }\end{array}$} & \multicolumn{5}{|c|}{ pdv simples } \\
\hline & \multirow{2}{*}{\multicolumn{3}{|c|}{ pdv complexes }} & \multicolumn{2}{|c|}{ pdv hiérachiques } \\
\hline & & & & \multicolumn{2}{|c|}{ pdv relationnels } \\
\hline \multirow{8}{*}{$\begin{array}{l}\text { Êtres discursifs, } \\
\text { ê-d }\end{array}$} & \multirow{3}{*}{ Première personne } & \multicolumn{2}{|c|}{$\begin{array}{l}\text { Le locuteur de } \\
\text { l'énoncé }\end{array}$} & \multicolumn{2}{|l|}{$1_{0}$} \\
\hline & & \multicolumn{2}{|c|}{ Locuteurs d'énoncé } & \multicolumn{2}{|l|}{$l_{t}(t \neq 0)$} \\
\hline & & \multicolumn{2}{|c|}{ Locuteurs textuels } & \multicolumn{2}{|l|}{$\mathrm{L}$} \\
\hline & \multirow[t]{2}{*}{ Deuxième personne } & \multicolumn{2}{|c|}{$\begin{array}{l}\text { Allocutaires } \\
\text { d'énoncé }\end{array}$} & \multicolumn{2}{|l|}{$a_{t}(t \neq 0)$} \\
\hline & & \multicolumn{2}{|c|}{ Allocutaires textuels } & \multicolumn{2}{|l|}{ A } \\
\hline & \multirow{3}{*}{ Troisième personne } & \multirow{2}{*}{\multicolumn{2}{|c|}{ Tiers individuels }} & Tiers d'énoncé & $\tau_{\mathrm{t}}(\mathrm{t} \neq 0)$ \\
\hline & & & & Tiers textuels & $\mathrm{T}$ \\
\hline & & Tiers & llectifs & \multicolumn{2}{|c|}{$\begin{array}{l}\text { Hétérogènes }(\mathrm{ON}) \leftrightarrow \text { Homogènes } \\
(\mathrm{LOI})\end{array}$} \\
\hline \multirow{3}{*}{$\begin{array}{l}\text { Liens } \\
\text { énonciatifs }\end{array}$} & \multicolumn{5}{|c|}{ Liens de responsabilité } \\
\hline & \multirow{2}{*}{\multicolumn{2}{|c|}{ Liens de non-responsabilité }} & Liens de non & -réfutation & \\
\hline & & & Lien de réfu & ation & \\
\hline
\end{tabular}

C'est le schéma théorique maximal. Il reste encore à montrer l'existence de plusieurs types au niveau du codage.

\section{Analyse polyphonique de quelques exemples}

Les éléments essentiels de la ScaPoLine introduits, il est temps d'appliquer la théorie à quelques exemples.

\subsection{Modalités}

Par modalité j'entends ici les formes et expressions linguistiques qui expriment l'attitude (au sens large) du locuteur par rapport à ce qu'il énonce (Nølke 2003 :183). Ainsi définies, les modalités introduisent des points de vue hiérarchiques. La structure générale d'un énoncé modalisé sera $M(p)$, où $M$ symbolise la modalité et $\mathrm{p}$ le contenu modalisé. Dès lors, l'analyse polyphonique générale de cette structure sera :

$$
\begin{aligned}
& \mathrm{M}(\mathrm{p}) \\
& \operatorname{pdv}_{1}:[\mathrm{X}](\mathrm{JUGE}(\mathrm{p})) \\
& \operatorname{pdv}_{2}:\left[1_{0}\right]\left(\mathrm{M}\left(\mathrm{pdv}_{1}\right)\right)
\end{aligned}
$$

$[\mathrm{X}]$ indique que la structure-p ne renferme aucune instruction relative à la saturation de la source de $\mathrm{pdv}_{1}$. Pour une modalité particulière, les composantes sont susceptibles d'être mieux cernées, et $\mathrm{M}$ recevra une analyse détaillée. On remarquera que le locuteur de l'énoncé, $1_{0}$, est source de la modalité par défaut. Cette valeur par défaut est particulièrement forte si $\mathrm{pdv}_{2}$ est soumis à la monstration. Peut-être nous en fournit un exemple ${ }^{14}$ :

\subsubsection{Peut-être}

En appliquant à peut-être l'analyse générale des modalités, nous aurons la description suivante :

(15) Peut-être (p) 


\author{
$\operatorname{pdv}_{1}:[\mathrm{X}](\mathrm{VRAI}(\mathrm{p}))$ \\ $\operatorname{pdv}_{2}:\left[1_{0}\right]\left(\right.$ PEUT-ÊTRE $\left.\left(\operatorname{pdv}_{1}\right)\right)$ \\ $\mathrm{X}=\mathrm{L}$ par défaut \\ PEUT-ÊTRE signifie :
}

- $1_{0}$ n'a pas de preuve ni en faveur de $p$ ni en faveur de non- $p$

- $1_{0}$ est conscient du fait que $\mathrm{X}$ a apparemment une preuve (un argument) en faveur de $p$

- $1_{0}$ accepte l'orientation discursive (argumentative) que $\mathrm{X}$ attache à $p$

- LOC construit $\mathrm{pdv}_{2}$ par monstration

Ce qui caractérise peut-être, ce sont les types de jugements qu'il invoque, ainsi que le fait que X soit le locuteur textuel par défaut. On notera que l'analyse logique «classique» ne rend compte que de la première propriété du jugement PEUT-ÊTRE. Or un exemple simple suffit à montrer que peut-être véhicule aussi les autres instructions :

(16) Pierre viendra peut-être demain.

Si quelqu'un énonce (16), il prévoit la venue de Pierre plutôt que sa non-venue, ce qui se révèle dans les enchaînements naturels :

(17) a. (16), ... il vaut donc mieux mettre une assiette de plus.

b. $(16), \ldots *$ nous pouvons donc enlever son assiette.

L'enchaînement dans (17b.) apparaît en effet déviant, ce qui s'explique par le fait que $1_{0}$ accepte l'orientation argumentative de 'Pierre viendra demain': sauf indication du contraire, il prévoit les conséquences de la venue de Pierre plutôt que celles de sa non-venue.

La quatrième propriété mentionnée a pour conséquence que l'adverbe peut-être ne supporte pas la focalisation simple : il ne peut ni être sous la négation, ni être focus du clivage, ni être dernier élément tonique de l'énoncé, ni être focus d'une question, ...

Il est important de souligner que L est seulement la valeur par défaut de X. La structure concessive étudiée dans 3.7. montre que d'autres êtres discursifs sont susceptibles de saturer la source :

(13) Peut-être que tu es resté chez toi toute la nuit, mais je t'ai vu au café à $22 \mathrm{~h}$.

Dans (13), X ne peut pas être L parce que p est concédé, c'est plutôt A (ou $a_{t}$ ). (13) montre aussi qu'accepter l'orientation argumentative ne veut dire accepter les conséquences qu'en défaut d'indication du contraire. En effet, le propre de la structure concessive est précisément d'indiquer que les conséquences prévues n'ont pas eu lieu. Il est d'ailleurs intéressant de noter que l'antéposition de peutêtre $\mathrm{a}$ une forte tendance à nous faire prévoir une concession ${ }^{15}$.

\title{
4.1.2 II semble que
}

Considérons maintenant brièvement la modalité Il semble que, qui, contrairement à peut-être est soumise à la véridiction: il est dit au sens de Wittgenstein (1961: §4.022sv). J'ai proposé ailleurs l'analyse suivante de cette modalité (Nølke $2001: 23)^{16}$ :

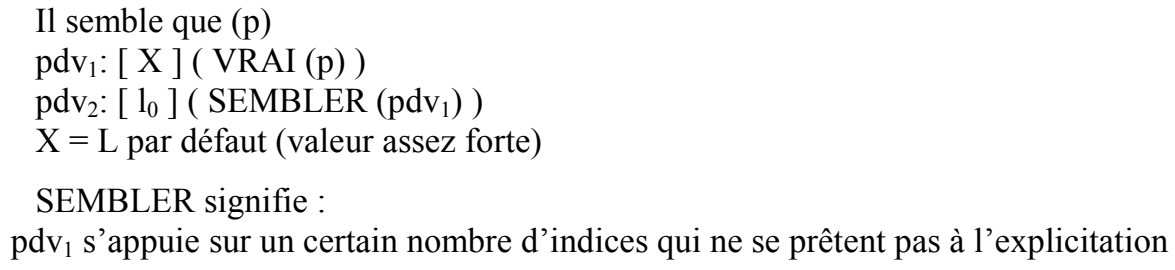

On remarquera que, dans la lecture par défaut, qui est presque obligatoire, il semble que introduit la polyphonie interne au sens strict, ce qui explique sans doute sa corrélation avec le subjonctif. Cette modalité introduit donc une sorte de discours intériorisé. 
Prenons un exemple simple pour illustrer l'analyse :

$$
\text { Il semble que Marie soit malade }
$$

Le fait que le locuteur de l'énoncé assume la responsabilité du discours intériorisé ( $\left.\mathrm{pdv}_{2}\right)$ entraîne que ce discours a lieu au moment de la parole. Cette analyse sera corroborée par une étude de la distribution de $i l$ semble que p (Nølke 2001 : 28sv.). Il découle par ailleurs du fait que L s'associe par défaut à pdv ${ }_{1}$ que $1_{0}$ accorde ce point de vue. Une autre conséquence en est que le locuteur peut enchaîner sur ce point de vue dans son discours continu. Témoin :

(20) Il semble que Marie soit malade. On ne pourra donc pas compter sur elle pour nous aider.

D'autre part, le fait qu'il ne s'agisse là que d'une valeur par défaut explique l'existence d'énoncés tels que (21):

(21) Il semble qu'on vive dans une démocratie, et voilà ce qui se passe.

qui est acceptable seulement parce que l'enchaînement fait entendre que $1_{0}$ réfute pdv ${ }_{1}$ d'où il suit que $\mathrm{L}$ n'assume pas la responsabilité de ce pdv. En effet, le locuteur de l'énoncé ne peut jamais réfuter un pdv dont le locuteur textuel assume la responsabilité (sauf au cas de schizophrénie !).

La modalité il semble que est particulièrement intéressante en ce qu'elle peut indiquer le passé du fait qu'elle comprend une forme verbale :

(22) Il semblait que Marie soit malade.

Dans ce cas, $l_{0}$ ne peut pas être tenu responsable de $\mathrm{pdv}_{2}$, car il n'existe qu'au moment de la parole. Néanmoins, tout porte à penser que l'analyse est fondamentalement valable même dans ce cas, car on a nettement l'impression que l'énoncé de (22) introduit, lui aussi, un discours intériorisé. La prise en considération des exemples avec le verbe au passé me mène donc à modifier un peu l'analyse proposée dans (18). Il suffit cependant de remplacer $l_{0}$ par $l_{t}$ où $t$ indique le moment d'évènement marqué par le verbe. L'analyse de Il semble que devient ainsi un argument en faveur de l'introduction des locuteurs d'énoncés ${ }^{17}$.

\subsection{Connecteurs}

Par connecteurs j'entends ici les formes et expressions linguistiques qui combinent des unités sémantiques - les arguments dudit connecteur - pour créer un nouveau sens complexe à partir de leurs sens plus primitifs en combinant et en spécifiant ceux-ci. Ainsi définis, les connecteurs introduisent des points de vue relationnels. La structure sémantique générale créée par un connecteur (prototypique) sera $p$ $C O N q$, où $p$ et $q$ symbolise les arguments et CON le connecteur.

Je considérerai deux connecteurs très utilisés : donc qui garde l'orientation discursive et mais qui la renverse et qui, partant, code une structure-p plus complexe que donc. Mes analyses s'appuient sur les nombreuses études publiées auxquelles ces deux connecteurs ont été soumis.

\subsubsection{Donc $^{18}$}

Comme toute une série de connecteurs, donc implique une relation de cause à effet. Dans son emploi « canonique », dont j'ai présenté une première analyse en 3.5, il s'appuie directement sur cette relation. Donc ne présente cependant pas la relation causale directement : sa fonction (logico-)sémantique se fonde sur un raisonnement. Cela ressort de la possibilité des deux énoncés suivants :

(8) Il fait beau, donc Pierre se promène. (Emploi « canonique » analysé dans 3.5)

(23) Pierre se promène, donc il fait beau.

Dans les deux énoncés, donc introduit une conséquence de ce qui précède (l'antécédent). Or cette conséquence n'est pas de type causal : elle est trouvée par un raisonnement. Notons que dans une lecture 
normale (vu notre conception du monde), l'interprétation des deux énoncés suppose la même idée préalable, à savoir que c'est le beau temps qui est la cause de la promenade de Pierre. Dans cette lecture, le raisonnement de (8) se base sur la déduction, tandis que celui de (23) applique l'abduction. C'est la possibilité de construire un raisonnement basé sur l'abduction qui distingue donc d'un connecteur comme de ce fait, par exemple :

a. Il fait beau, de ce fait Pierre se promène.

b. : ? Pierre se promène, de ce fait il fait beau.

(24b.) est acceptable seulement dans une lecture où c'est la promenade de Pierre qui déclenche le beau temps. De ce fait introduit en effet (directement) une relation causale entre faits plutôt qu'un raisonnement, et l'abduction est exclue.

Si nous symbolisons le beau temps par $p$ et la promenade de Pierre par $q$, nous pourrons proposer une analyse polyphonique révisée de donc :

$\mathbf{p d v}_{\mathbf{1}}$ : [ X ] ( VRAI (la proposition, $p$ ou $q$, véhiculée par l'antécédent) )

$\mathbf{p d v}_{\mathbf{2}}:\left[\mathrm{l}_{0}\right]$ ( VRAI (la proposition, $p$ ou $q$, véhiculée par le conséquent) )

$\operatorname{pdv}_{\mathbf{3}}:\left[1_{0}\right]($ TOP $(\operatorname{si} p$ alors $q))$

$\mathrm{L}$ est responsable de $\mathrm{pdv}_{1}$ par défaut

Rappelons que TOP signifie 'il est généralement vrai que'.

Une très large gamme de connecteurs font intervenir un raisonnement et introduisent des topoï de cette manière. Nous dirons que ces connecteurs impliquent une logique d'inférences :

\section{La logique d'inférences}

L'ensemble des topoï appliqués dans un discours particulier constitue une logique d'inférences associée à un être discursif. Cet ê-d est appelé le raisonneur.

Le locuteur de l'énoncé est raisonneur par défaut, car les relations logiques se fondent le plus souvent sur la logique du locuteur qui présente son raisonnement hic et nunc. Il peut cependant s'agir de n'importe quel autre ê-d construit par LOC. Cela arrive souvent dans le discours représenté (ou rapporté) où il est souvent difficile de voir qui est raisonneur. Prenons un exemple :

(25) Elle confondait, dans son désir, les sensualités du luxe avec les joies du cœur, l'élégance des habitudes et les délicatesses du sentiment. Ne fallait-il pas à l'amour, comme aux plantes indiennes, des terrains préparés, une température particulière ? Les soupirs au clair de lune, les longues étreintes, les larmes qui coulent sur les mains qu'on abandonne, toutes les fièvres de la chair et les langueurs de la tendresse ne se séparaient donc pas du balcon des grands châteaux qui sont pleins de loisirs, d'un boudoir à stores de soie avec un tapis bien épais, des jardinières remplies, un lit monté sur une estrade, ni du scintillement des pierres précieuses et des aiguillettes de la livrée.

(Madame Bovary : I,ix ; p. 55) (le soulignement de donc est de moi)

Dans ce texte, donc se trouve dans un segment de discours représenté introduit par la question rhétorique Ne fallait-il pas.... Dès lors, la question se pose de savoir qui est raisonneur. Le lecteur attentif de Madame Bovary sait que le topos invoqué n'appartient pas à la logique d'inférences du locuteur. Il ne reste donc que l'interprétation selon laquelle c'est la logique du personnage (Emma en l'occurrence). L'ironie de ce texte découle alors du fait que le topos activé n'est pas accepté par l'auteur (et peut-être non plus par l'opinion générale, c'est-à-dire par ON). Il semble que l'ironie célèbre de Flaubert a souvent recours à ce procédé polyphonique.

\subsubsection{Mais}

Il n'y a guère de connecteur français plus étudié que mais. Dans ma proposition d'analyse ScaPoLine, je m'appuie sur l'analyse d'Anscombre et Ducrot, qui a acquis droit de cité. Selon cette analyse, mais 
implique trois arguments dont un est (le plus) souvent sous-entendu. Illustrons l'analyse par un exemple simple :

(26) Pierre est riche mais il est avare.

En symbolisant 'Pierre est riche' par $p$ et 'Pierre est avare' par $q$, nous aurons la structure sous (27) :

$$
\begin{aligned}
& p \rightarrow r \\
& q \rightarrow \text { non } r \\
& p \text { MAIS } q \rightarrow \text { non } r
\end{aligned}
$$

où ' $\rightarrow$ ' signifie 'est un argument en faveur de', et où $r$ est une conclusion qu'il faudra déduire du contexte et/ou de la situation.

Réinterprétée dans le cadre de la ScaPoLine, je propose l'analyse polyphonique suivante de mais :

\section{(28) Fonction sémantico-logique de mais}

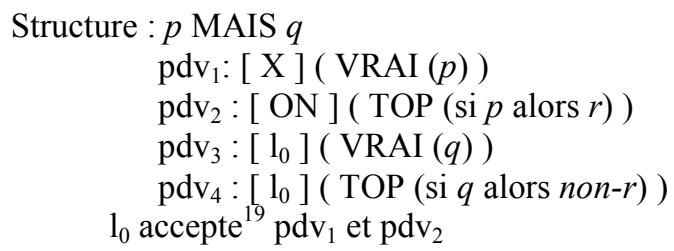

Pour comprendre (26) il faudra trouver ou inventer $r$. Disons que $r$ serait 'Pierre est susceptible de nous donner de l'argent si nous le demandons'. L'analyse sous (28) exprime alors que quelqu'un (peut-être de non repérable), $\mathrm{X}$, considère qu'il est vrai que Pierre est riche. Le locuteur de l'énoncé est d'accord. Puis, selon l'opinion générale, $\mathrm{ON}$, c'est normalement le cas qu'une personne riche est prête à donner de l'argent si on lui demande. Le locuteur textuel, L, fait partie de ce $\mathrm{ON}$, d'où il suit que $\mathrm{l}_{0}$ accorde le pdv de $\mathrm{ON}^{20}$. Le locuteur de l'énoncé affirme que Pierre est (aussi) avare et il avance qu'il est généralement vrai que les personnes avares ne donnent pas de l'argent même si on leur demande. Le résultat de cette structure (concessive) complexe est que (26) fournit un argument en faveur de laisser tomber l'idée (éventuelle) de demander de l'argent à Pierre.

Cet exemple simple montre à quel point le petit connecteur mais peut introduire tout un discours et à quel point il faut connaître le contexte pour avoir une chance de comprendre l'intention du locuteur. Dans ces conditions, il n'est guère étonnant que ce connecteur, d'une innocence qui n'est qu'apparente, constitue une arme forte dans l'arsenal linguistique d'un locuteur habile. Prenons à titre d'exemple un texte qui provient de la plume de Flaubert, qui maîtrisait les subtilités linguistiques mieux que personne :

(29) Comme elle [Emma] fut longtemps avant de trouver son étui son père s'impatienta; elle ne répondit rien; mais tout en cousant elle se piquait les doigts qu'elle portait ensuite à sa bouche pour les sucer.

Charles fut surpris de la blancheur de ses ongles. [...]

(Flaubert $1971: 38-39$ )

Admettons que $p$ soit 'elle ne répondit rien', ou peut-être plutôt 'elle se taisait', et que $q$ soit 'elle se piquait les doigts'. Quel pourrait être $r$ alors ? Pour trouver cet argument implicite du connecteur, il faudra faire appel au contexte. Un locuteur moderne non initié, qui ne dispose d'aucune connaissance du contexte dans lequel le roman fut écrit, ne peut recourir qu'au cotexte. Il pourrait proposer que $r$ soit 'il n'y a aucune raison de faire attention à Emma', ce qui rendrait la continuation plausible, mais cette lecture ne rendrait guère justice au texte. Les topoï invoqués dépendent en effet directement des idées reçues de l'époque. Nul doute qu'un lecteur contemporain a facilement compris qu'il y a dans cet exemple tout un discours intérieur d'Emma qui est rapporté par l'emploi de mais dans ce contexte. En même temps, ce passage raconte un moment clé du roman : Charles tombe amoureux d'Emma ${ }^{21}$. 


\section{Conclusions}

J'espère avoir montré que l'appareil formel introduit nous permet de préciser des nuances de sens assez fines et que la méthodologie nous sert de guide dans notre tentative de révéler, pour chaque élément linguistique, l'apport de la forme linguistique à la genèse du sens. Par l'analyse de quelques fragments de textes littéraires, j'ai ensuite tenté de faire apparaître que la ScaPoLine devient ainsi pour les analyses textuelles un outil opérationnel qui peut servir à améliorer notre compréhension des textes.

Il s'agit toutefois d'une théorie en évolution constante. Non seulement beaucoup de phénomènes linguistiques n'ont pas encore été examinés, mais il reste aussi bien des problèmes épineux à résoudre quant à la structuration interne de la ScaPoLine. Pour n'en mentionner que quelques-uns : quel est le rapport entre la composante JUGE des pdv et les liens énonciatifs ? Combien de liens différents de nonresponsabilité et de non-réfutation existe-t-il, et dans quelle mesure sont-ils susceptibles d'être marqués par les matériaux linguistiques? Comment s'articulent les rapports entre les différents (types de) pdv? Tous les types d'êtres discursifs théoriquement possibles sont-ils empiriquement pertinents ?

Pour moi, les perspectives qu'ouvre la ScaPoLine l'emportent cependant largement sur les problèmes. Située dans un cadre modulaire (Nølke 1994), la théorie invite à la collaboration et se prête à des recherches interdisciplinaires, non seulement entre linguistes venant de différentes écoles mais aussi entre chercheurs venant de différentes sciences. Voilà pour moi l'argument le plus important pour continuer la recherche polyphonique. Que toutes voix se joignent pour la grande polyphonie du savoir !

\section{Références bibliographiques}

Anscombre, J.-C. (2005). Le ON-locuteur : une entité aux multiples visages. in Bres J. et al. (éds).

Bakhtine, M. (1970). La Poétique de Dostoïevski. Paris : Seuil.

Banfield, A. (1979). Où l'épistémologie, le style et la grammaire rencontrent la théorie littéraire. Langue française, 44, 9-26.

Berrendonner, A. (1981). Éléments de pragmatique linguistique. Paris : Éditions de Minuit.

Bres, J. (2005). Savoir de quoi on parle : dialogue, dialogal, dialogique ; dialogisme, polyphonie.... in : Bres J. et al. (éds.). 47-61.

Bres, J., Haillet, P., Mellet, S., Nølke, H., Rosier, L. (éds) 2005. Dialogisme, polyphonie : approches linguistiques. Bruxelles : Duculot.

Coltier, D. \& Dendale, P., 2005, «La notion de prise en charge ou responsabilité dans la théorie scandinave de la polyphonie linguistique ", in Bres J. et al. (éds).

Dendale, P. (2007). A critical survey and comparison of French and Scandinavian frameworks for the description of linguistic polyphony and dialogism. in: Therkelsen, R. et al. (éds). 109-143.

Désclés, J.-P. (1976). Quelques opérations énonciatives. in: Logique et niveaux d'analyse linguistique. Paris: Klinsieck. 213-242.

Ducrot, O. (1972). Dire et ne pas dire. Paris, Hermann.

Ducrot, O. (1982). La notion de sujet parlant. Recherches sur la pholosophie et le langage, Université de Grenoble. 65-93.

Ducrot, O. (1984). Le dire et le dit. Paris : Minuit.

Ducrot, O. (2001). Quelques raisons de distinguer «locuteurs» et «énonciateurs ». Polyphonie - linguistique et littéraire, III, 19-41.

Fauconnier G. (1984). Espaces mentaux, Paris, Minuit.

Flaubert, G. (1971). Madame Bovary, éd. Class. Paris : Garnier.

Fløttum, K. (2001). Les liens énonciatifs : tentative d'une nouvelle typologie. Polyphonie - linguistique et littéraire, III, 67-86. 
Genette, G. (1972). Figures III, Paris, Seuil.

Jønsson, M. (2004). En polyfon analyse af udsigelsesadverbialerne. Sproglig polyfoni, II. 97-126

Kratschmer, A. (2007). Empirisk evidens for historiske og tredjepersonsdiskursindivider: italienske kontekster med sembrare, parere i datid. in: Therkelsen, R. et al. (éds). 325-343.

Nølke, H. (1983). Les adverbes paradigmatisants : fonction et analyse. Revue Romane Numéro Spécial 23. Copenhague : Akademisk Forlag.

Nølke, H. (1985). Le subjonctif : fragments d'une théorie énonciative. Langages 80. 55-70.

Nølke, H. (1993). Le regard du locuteur. Pour une linguistique des traces énonciatives. Paris : Kimé.

Nølke, H. (1994) Linguistique modulaire : de la forme au sens. Louvain/Paris : Peeters.

Nølke, H. (2001). Le regard du locuteur 2. Pour une linguistique des traces énonciatives. Paris : Kimé.

Nølke, H. (2003). Modalité(s) énonciative(s) adverbiale(s). in : Birkelund et al. (éds). Aspects de la modalité. Tübingen : Niemeyer. 181-192.

Nølke, H. (2005). Le locuteur comme constructeur du sens. in : Bres J. et al. (éds). 111-124.

Nølke, H. (2006a). Pour une théorie linguistique de la polyphonie : problèmes, avantages, perspectives, in : L. Perrin (éd) : Le sens et ses voix. Dialogisme et polyphonie en langue et en discours. Metz: Université Paul Verlaine. 243-269.

Nølke, H. (2006b). Connecteurs pragmatiques: l'apport de quelques connecteurs à la structure polyphonique. Français Moderne, 1, 32-42.

Nølke, H., Fløttum, K. \& Norén, C. (2004). ScaPoLine. La théorie scandinave de la polyphonie linguistique. Paris : Kimé.

Perrin, L. (2003). Citation, opacité, point de vue. Polyphonie - linguistique et littéraire, VII, 63-93.

Plénat, M. (1979). Sur la grammaire du style indirect libre. Cahiers de grammaire, 1, 95-140.

Rabattel, A.(2003). La (pré-)réflexivité dans les comptes rendus de perceptions, de paroles et de pensées : la question du mimétisme dans les discours représentés. Polyphonie - linguistique et littéraire, VII, 1-31.

Roulet, E., Filliettaz, L., Grobet, A. (2001). Un modèle et un instrument d'analyse de l'organisation du discours. Berne : Peter Lang.

Therkelsen, R., Andersen, N. M., Nølke, H. (éds) (2007). Sproglig polyfoni. Tekster om Bachtin \& ScaPoLine. Århus : Aarhus Universitetsforlag.

Wittgenstein, L. (1961). Tractatus Logico-philosophicus. Londres : Routledge \& Kegan Paul.

\footnotetext{
${ }^{1}$ En fait, on trouve les premières traces d'une description polyphonique dans Ducrot $(1972: 38)$, déjà, lorsque cet auteur distingue négation descriptive et négation polémique. Ainsi dit-il à propos de la négation ne...pas dans l'exemple (7) Ce mur n'est pas blanc (désormais devenu l'exemple canonique de la polyphonie) que « La plupart du temps, on l'emploiera pour marquer qu'on s'oppose à une affirmation antérieure Ce mur est blanc (affirmation qui peut d'ailleurs n'avoir pas été formulée explicitement par le destinataire de (7), mais lui être seulement prêtée par le locuteur ».

${ }^{2}$ C'est là apparemment exactement la définition que propose la praxématique du dialogisme, terme qui semble plus fidèle aux travaux de Bakhtine (cf. Bres 2005).

${ }^{3}$ Il existe sans doute là une différence essentielle entre langue orale et langue écrite. A l'oral, l'énonciation est directement observable et influe ainsi sur l'interprétation de l'énoncé, tandis qu'à l'écrit, il est particulièrement important que l'énoncé ne soit rien d'autre qu'une image de l'énonciation. Il s'ensuit que les conditions interprétatives diffèrent considérablement entre les deux registres. A l'écrit, l'énoncé - et par là le codage linguistique - joue un rôle bien plus important qu'à l'oral. En ce sens, la ScaPoLine s'occupe principalement de la langue écrite se joignant là, me semble-t-il, aux autres théories sémantiques sur le marché. L'interaction entre les observations
} 
simultanées de l'énonciation et de l'énoncé qui a lieu à l'oral mériterait des études pointues qui pourraient nous renseigner davantage sur le poids qu'a le codage linguistique pour l'interprétation réelle. De nature proprement pragmatique, ces études sortiraient cependant du cadre de la ScaPoLine.

${ }^{4}$ Il nous arrive aussi de dire que la source assume la responsabilité du pdv ou qu'elle est tenue responsable du pdv, ou même que le pdv est assumé par elle.

${ }^{5}$ Cette idée a été développée dans Jønsson (2004).

${ }^{6}$ Voir par exemple Perrin (2003), qui associe toute espèce de discours rapporté à une forme d'imitation, de reproduction mimétique de ce qui a été dit. Voir aussi Rabattel (2003).

${ }^{7}$ Le discours indirect rapporté nous en fournit des exemples (p.ex. Pierre a dit qu'il viendrait).

${ }^{8}$ On en a un exemple simple dans l'énoncé : Pierre croit que la terre est ronde.

${ }^{9}$ Il est en effet pertinent pour l'analyse de la polyphonie codée de distinguer différentes instances de ON selon les éléments qu'il renferme, et notamment selon l'inclusion ou la non-inclusion de $\mathrm{L}$ et de A. Ainsi, la présupposition faible (Nølke 1983 : 33) ne fait pas intervenir A.

${ }^{10}$ Pour les arguments empiriques en faveur de cette sous-catégorisation, voir Fløttum (2001).

${ }^{11}$ On remarquera que ce modèle des liens diffère de celui que nous avons proposé dans Nølke, Fløttum \& Norén (2004). La différence s'explique par le fait que, dans notre ouvrage, nous avons insisté sur le fonctionnement textuel; ici en revanche, je ne considère que le niveau de l'énoncé.

${ }^{12}$ Peut-être y a-t-il même deux échelles, l'une orientée vers la responsabilité, l'autre vers la réfutation. Cette question attend encore des analyses empiriques.

${ }^{13}$ Pendant très longtemps les notions de responsabilité et de prise en charge ont été confondues dans le cadre de la ScaPoLine. C'est grâce aux analyses critiques comparatives très détaillés effectuées par Coltier \& Dendale (2005) que j'ai été amené à préciser notre notion de responsabilité de sorte qu'elle se distingue nettement de celle de prise en charge, qui, d'ailleurs, n'a jamais eu de statut formel dans la théorie, mais dont je me suis souvent servi de manière informelle, ce qui a semé pas mal de confusion.

${ }^{14}$ Je reprends et reformule ici l'analyse de peut-ètre proposée dans Nølke (1993). Voir ce texte pour des analyses de toute une gamme d'emplois différents de cet adverbe.

${ }^{15}$ Ce phénomène a été creusé dans Nølke (1993).

${ }^{16}$ Je reprends et reformule ici l'étude de Il semble que présentée dans Nølke (2001 : 15-34).

${ }^{17}$ Kratschmer (2007) a étudié l'équivalent italien sembra che et a présenté une série d'arguments empiriques en faveur de l'existence d'êtres discursifs d'énoncé de la deuxième et de la troisième personne également. Ces études restent à faire pour le français, mais tout porte à croire que la situation française est tout à fait parallèle.

${ }^{18}$ Pour une analyse polyphonique poussée de donc voir Nølke (2006b).

${ }^{19}$ Plus précisément, il engage un lien de non-responsabilité et de non-réfutation avec $\operatorname{pdv}_{1}$.

${ }^{20}$ Selon une règle générale de la ScaPoLine, $1_{0}$ accepte un pdv dont $\mathrm{L}$ assume la responsabilité.

${ }^{21}$ Ce passage a été soumis à de nombreuses analyses littéraires. Il me semble que ces analyses auraient pu profiter de l'analyse linguistique de l'importance que joue mais pour l'interprétation. 